\title{
Clinico-pathological Study of Different Modalities of Surgery of Typhoid Perforation Treatment
}

\author{
Authors \\ Dr Mamun Ibn Munim', Dr Guljar Ahmed ${ }^{2}$, Dr Hasina Choudhury ${ }^{3}$ \\ Dr Mushfiqur Rahman ${ }^{4}$, Dr Moontaha Choudhury ${ }^{5}$ \\ ${ }^{1}$ Assistant Professor, North East Medical College, Sylhet \\ ${ }^{2}$ Associate Professor, North East Medical College, Sylhet \\ ${ }^{3}$ DGO; MS (Thesis Part-Gynae \& Obs), OSD (DG health) \\ ${ }^{4}$ Associate Professor, Prime Medical College, Rangpur \\ ${ }^{5}$ Indoor Medical Officer, Ragib-Rabeya Medical College, Sylhet
}

\begin{abstract}
Introduction: Small bowel perforation is a common surgical emergency all over the world .The spectrum of etiology of small bowel perforation differs from its western counterpart. Typhoid fever is a protracted disease that includes bacteremic phase with fever and chills during the first week, wide spread reticulo-endothelial involvement with rash, abdominal pain and prostration in the second week and ulceration of Payer's patches with intestinal bleeding and perforation during the third week.
\end{abstract}

Objective: The main goal of our study is to identify the suitable treatment of typhoid perforation by comparing different modalities of surgery.

Methodology: This cross-sectional observational study was conducted in the different units of Department of Surgery Sylhet MAG Osmani Medical College Hospital during 1st January 2010 to 31st December 2011. A total 63 patients with typhoid ulcer perforation were enrolled in this study.

Results: Out of 63 typhoid ulcer patients 4 (6.35\%) patients were below the age of 20 years. The mean age of this group of patients was $18.76( \pm 0.39) .32(50.79 \%)$ patients were found between the ages of $20-40$ years. Among them $48(76.19 \%)$ patients were male and $15(23.81 \%)$ patients were female. All 63 (100\%) patients presented with abdominal pain. Total number of complication exceeds the total number of patients as the more than one complication occurred in one patient. Complications occurred in $7(33.33 \%)$ patients who came to the hospital within 48 hours and $14(66.66 \%)$ patients in those whom came to the hospital after 48 hours of onset of symptoms. Total complication occurred in 21 patients. The association between time lapse in hospitalization and post-operative complication is statistically significant $(p<0.05)$. There is significant difference between the death of the patients who reached earlier in the hospital and who are not $(p<0.05)$. we can conclude that In case of early presentation repair of perforation with proximal ileostomy and in case of late presentation - resection, end to end anastomosis with proximal ileostomy have the better outcome.

Keyword: Small bowel perforation, Typhoid fever, Typhoid ulcer perforation.

\section{Introduction}

Gastrointestinal perforation also called ruptured bowel is a hole in the wall of part of the gastrointestinal tract. The gastrointestinal tract contains the esophagus, stomach, small intestine, and large intestine. Symptoms incorporate extreme stomach torment and tenderness. ${ }^{[1][2]} \mathrm{A}$ person who has a perforated bowel contain hole 
in the wall of the stomach, small intestine or large intestine. This perforated bowel allows food or stool to leak into the abdomen and because of that serious inflammation inside of the abdomen is caused by food or stool which is called peritonitis. Clinical records of 323 patients with typhoid fever were reviewed to study the incidence, fatality and optimal therapy of the complication of intestinal perforation in Bangladesh where Fifteen patients (4.6\%) developed intestinal perforation. Perforation of the small intestine results in the potential for bacterial contamination of the abdominal cavity. Peritonitis following perforation of small intestine may lead to multiorgan failure and death unless it is treated promptly and vigorously and surgical emergency.
Typhoid fever and paratyphoid fever is a systemic infection caused by Salmonella enterica, including S. enterica serotype. Intestinal bleeding in typhoid fever usually occurs from the ulcers in the ileum or the proximal colon, and the most common colonoscopic manifestations are multiple variablesized punched-out ulcerations. This Typhoid ulcer perforation presents as complication of typhoid ulcer. Person with Typhoid ulcer perforation typically presents as sudden severe abdominal pain, muscle guard, rigidity, tenderness of the whole abdomen, rebound tenderness and absent bowel sound with a background of fever for 7 to 10 days also may have toxic, hypotensive, dehydrated. Small gut perforation must be managed by surgically ${ }^{[3][4]}$
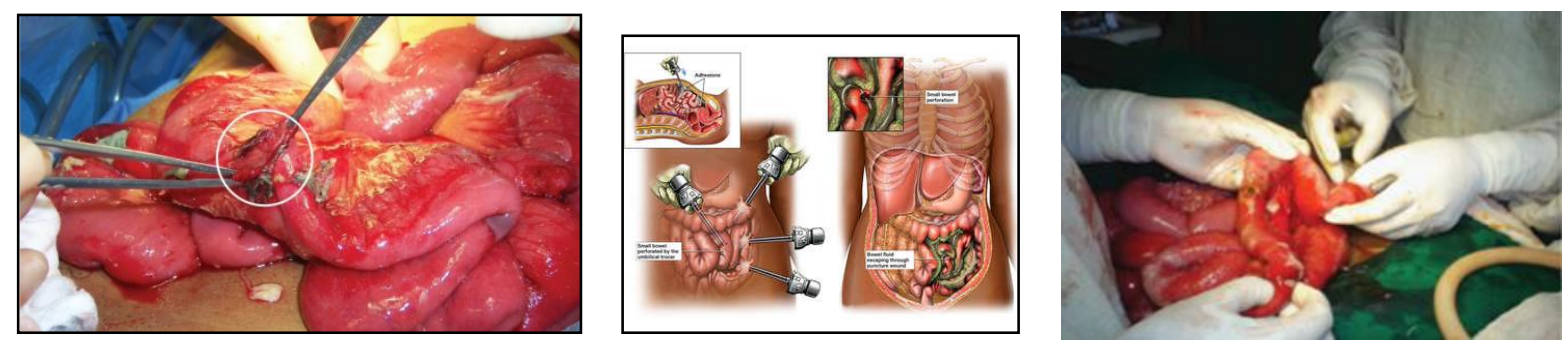

Figure-1a,1b,1c: During surgery of small and typhoid ulcer perforation. ${ }^{[5]}$

Depending upon the site of perforation, cause of the perforation and condition of the patient there are different modalities of surgery. Surgical modality includes primary repair; repair and ileostomy; resection and ileocolic anastomosis; resection and end to end anastomosis; resection, anastomosis and proximal ileostomy. In all cases there must be peritoneal toileting. In this study our optimum objective is to evaluate the efficiency of different modalities of surgery of typhoid perforation by compare their outcome.

\section{Objective}

\section{General Objective}

To identify the efficacy rate of different modalities of surgery of typhoid perforation by compare their outcome.

\section{Specific Objective}

$>$ To observe the presentations of the patients of typhoid perforation.

$>$ To record the time of 1 st symptom and time of treatment.

$>$ To identify the treatment modalities of the individual patient.

$>$ To examine complications and mortality of typhoid perforation.

$>$ To evaluate outcome of treatment according to time lapse before hospitalization.

\section{Methodology}

Study type

$>$ This study was a cross-sectional observational study.

\section{Study Place and time}

$>$ This study was conducted in different units of Department of Surgery Sylhet MAG 
Osmani Medical College Hospital, Sylhet from 1st January 2010 to 31st December 2011 with diagnosis of typhoid ulcer perforation, were taken as target population.

\section{Inclusion Criteria}

$>$ Patients with typhoid ulcer perforation underwent operative treatment.

$>$ Age of the patients were 18 years or above.

$>$ Both sexes.

\section{Exclusion Criteria}

$>$ Traumatic perforation.

$>$ Tubercular ulcer perforation.

$>$ Perforation other than small gut.

$>$ Diabetes mellitus.

$>$ Renal failure.

$>$ Debilitated patient.

$>$ Malignancy.

$>$ Patients failing to come for follow-up of up to 30 days since the day of operation.

\section{Sample Size}

Causes of small bowel perforation are various. Among them outcome of typhoid ulcer perforation is worst. Incidence of typhoid ulcer perforation is taken as unit for calculation of sample size. Sample size was calculated by using Guilford and Frucher formula considering $5 \%$ level of significance, $5 \%$ precision level (marginal error) and considering the $4.6 \%$ incidence of typhoid ulcer perforation. Calculated sample size was 68.Due to time limitation and non availability of case 63 cases are taken as sample size in this study.

\section{Method of Data Collection}

$>$ During the study data was collected by both quantitative and qualitative method by using pre designed questionnaire designed for the study. The questionnaire was pre tested and face validated by consulting experts. The patient was taken to the operation theatre after taking informed consent and improvement of homodynamic status (Pulse$100 /$ min or less, systolic blood pressure $>100$ $\mathrm{mmHg}$ and urine output $>30 \mathrm{ml} /$ hour). After opening of the abdomen intra-peritoneal exudates were sucked out and site of perforation were searched. Numbers of perforations were also looked for. Type of peritoneal exudes were looked whether they were purulent, fibrinous or serosenguinous. Further procedures were done according to the site of perforation, number of the perforation, condition of the gut and general condition of the patient. Proper haemostasis was done with diathermy coagulation or ligated the bleeding vessel. Every effort was made to protect the wound margin from contamination when dealing with any other source of peritoneal contaminations.

\section{Statistical Analysis}

$>$ Data were processed and analyzed with both manually and using SPSS Version 16.0.

$>$ Quantitative data summarized by mean and standard deviation.

$>$ Qualitative data summarized by ratio and percentage.

$>$ Comparison done by $\mathrm{x} 2$ test for association. $\mathrm{P}<0.05$ considered as significant, $\mathrm{p}>0.05$ considered as non significant and $\mathrm{p}<0.01$ considered as highly significant.

\section{Result}

Out of total 519 cases of suspected gut perforation, 68 cases giving the incidence rate of typhoid ulcer perforation $4.6 \%$ which is shown by Butler et al. Due to time limitation, 63 cases are included in this study fulfilling the enrollment criteria. In table-1 shows Distribution of age of the Patients where Out of 63 typhoid ulcer patients 4 $(6.35 \%)$ patients were below the age of 20 years. The mean age of this group of patients was 18.76 $( \pm 0.39) .32(50.79 \%)$ patients were found between the ages of $20-40$ years. Mean age of this group of patients was $32.17( \pm 7.63)$. The following figure is given below: 
Table-1: Distribution of age of the Patients $(n=63)$

\begin{tabular}{|l|c|c|c|}
\hline Age group & Frequency & Percentage & Mean \\
\hline$<20$ years & 4 & 6.35 & $18.76( \pm 0.39)$ \\
\hline $20-40$ years & 32 & 50.79 & $32.17( \pm 7.63)$ \\
\hline $40-60$ years & 23 & 36.51 & $49.11( \pm 8.16)$ \\
\hline 60-80 years & 4 & 6.35 & $68.39( \pm 1.97)$ \\
\hline Total & $\mathbf{6 3}$ & $\mathbf{1 0 0}$ & \\
\hline
\end{tabular}

In figure-2 shows Distribution of sex of the of male and female patients was 3.2:1. The Patients where $48(76.19 \%)$ patients were male following figure is given below: and $15(23.81 \%)$ patients were female. The ratio

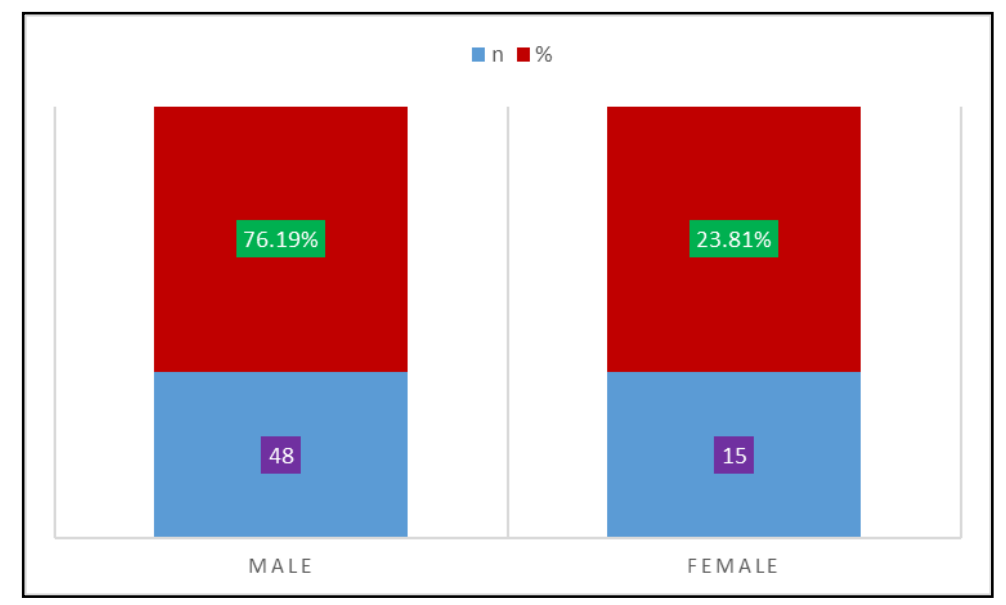

Figure-2: Distribution of sex of the Patients $(n=63)$

In Table-2 shows Distribution of Clinical Presentation of the Patients (major signs on admission) where All 63 (100\%) patients presented with abdominal tenderness, muscle guard, rigidity and rebound tenderness. Among the other sigs; $14(22.22 \%)$ patients had tachycardia, $7(11.11 \%)$ patients had hypotension,

Table-2: Distribution of Major Signs on Admission
$10(15.87 \%)$ patients had dehydration, 56 $(88.89 \%)$ patients had raised body temperature, 42 $(66.67 \%)$ patients had abdominal distension and $32(51 \%)$ patients had obliteration of liver dullness. Frequency exceeds 63 and percentage exceeds 100 due to more than one major signs in same patient. The following table is given below:

\begin{tabular}{|l|c|c|}
\hline Major signs & Frequency & percentage \\
\hline Tachycardia & 14 & 22.22 \\
\hline Hypotension & 7 & 11.11 \\
\hline Dehydration & 10 & 15.87 \\
\hline Raised body temperature & 56 & 88.89 \\
\hline Abdominal distension & 42 & 66.67 \\
\hline Abdominal tenderness & 63 & 100 \\
\hline Muscle guard \& rigidity & 63 & 100 \\
\hline Rebound tenderness & 63 & 100 \\
\hline Obliteration of liver dullness & 32 & 51 \\
\hline Absent bowel sound & 63 & 100 \\
\hline Total & 413 & 566 \\
\hline
\end{tabular}

In figure-3 shows Distribution of Ultrasonography Findings where $41.27 \%$ patients had intraperitoneal collection. The peritoneal cavities of $68.73 \%$ patients were free of intraperitoneal collection. The following table is given below: 


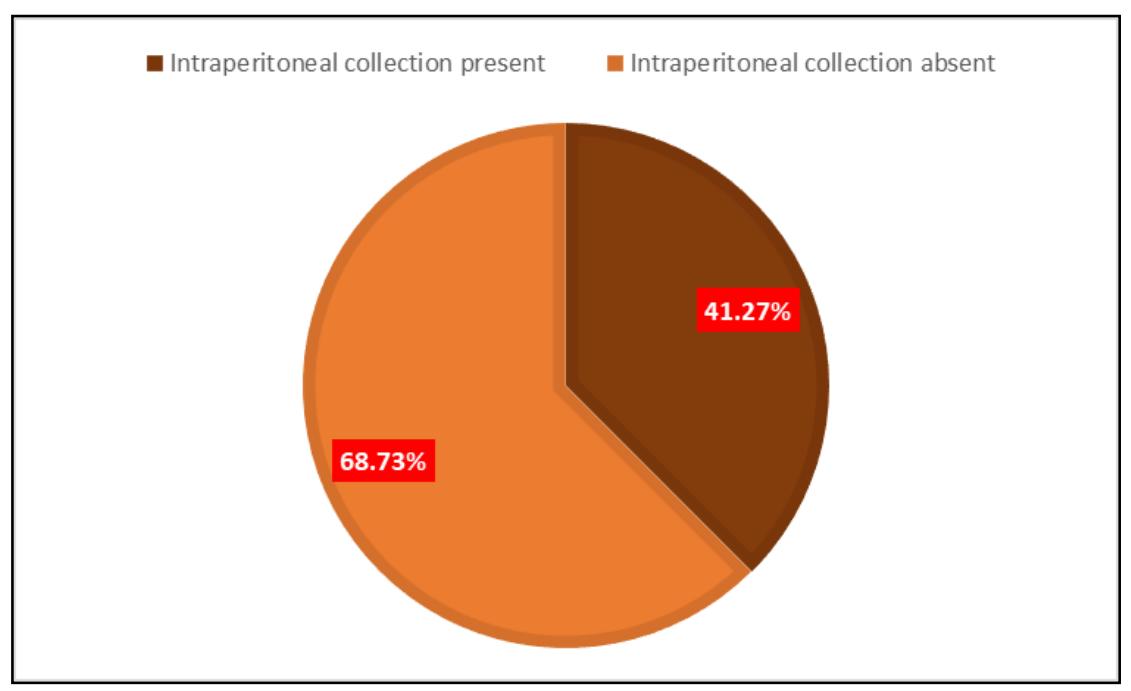

Figure-3: Distribution of Ultrasonography Findings

In table-4 shows Distribution by Collection in the Peritoneal Cavity where Peritoneal exudates were purulent in $33(52.78 \%)$ patients. Fibrinous exudates were found in peritoneal cavity of 26

Table-4 : Distribution by Collection in the Peritoneal Cavity

\begin{tabular}{|l|c|c|}
\hline Peritoneal exudates & Frequency & percentages \\
\hline Purulent & 33 & 52.78 \\
\hline Fibrinous & 26 & 41.27 \\
\hline Serosanguinous & 4 & 6.35 \\
\hline Total & 63 & 100 \\
\hline
\end{tabular}

In figure-4 shows Distribution by Morphology of the Typhoid Ulcer Perforation where Omental adhesion over the perforation was found in 3 patients, 40 patients had open perforation, in 11
$(41.27 \%)$ patients. In $4(6.35 \%)$ patients peritoneal cavity was full of serosanguinous fluid. The following table is given below:

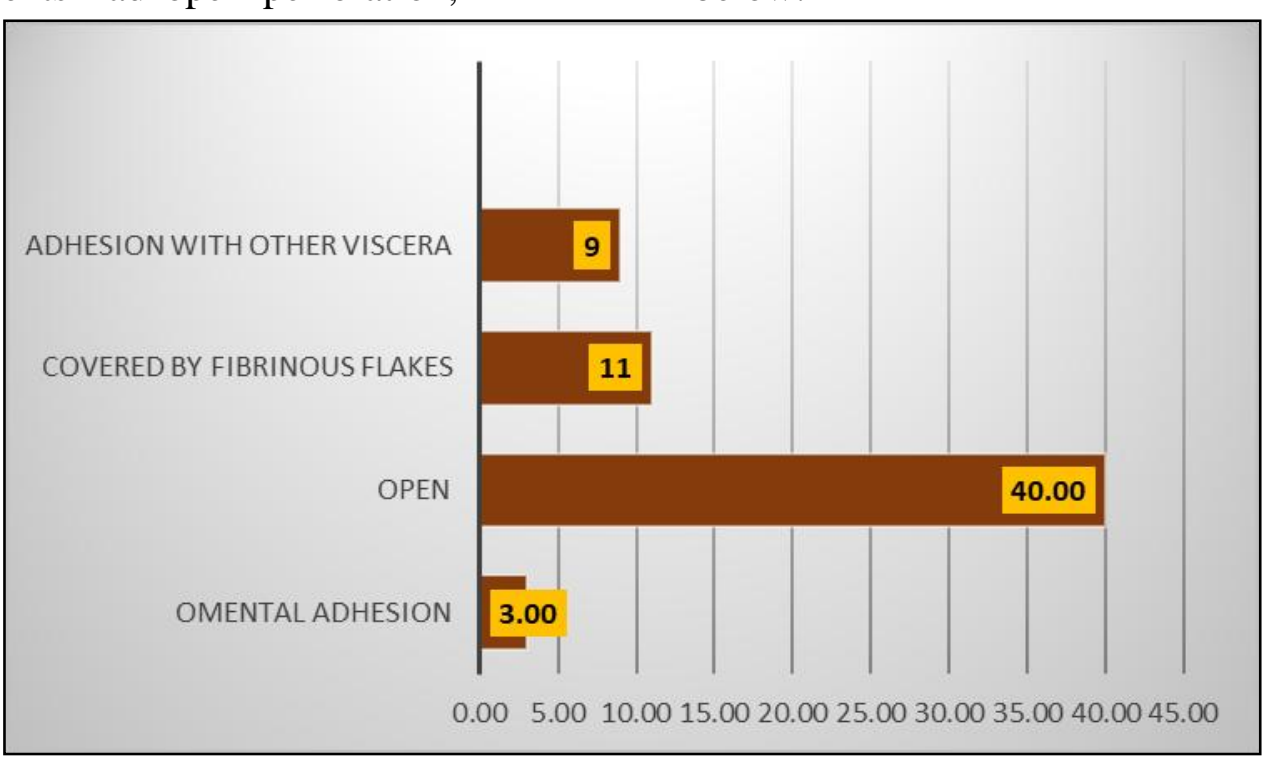

Figure-4: Distribution by Morphology of the Typhoid Ulcer Perforation

In Figure -6 shows Distribution by Number of perforation where among the 63 patients; $63.49 \%$ patients had single perforation and $36.51 \%$ patients patients perforation was covered by fibrinous flakes and 9 patients had adhesion of perforation with other viscera. The following figure is given below: 


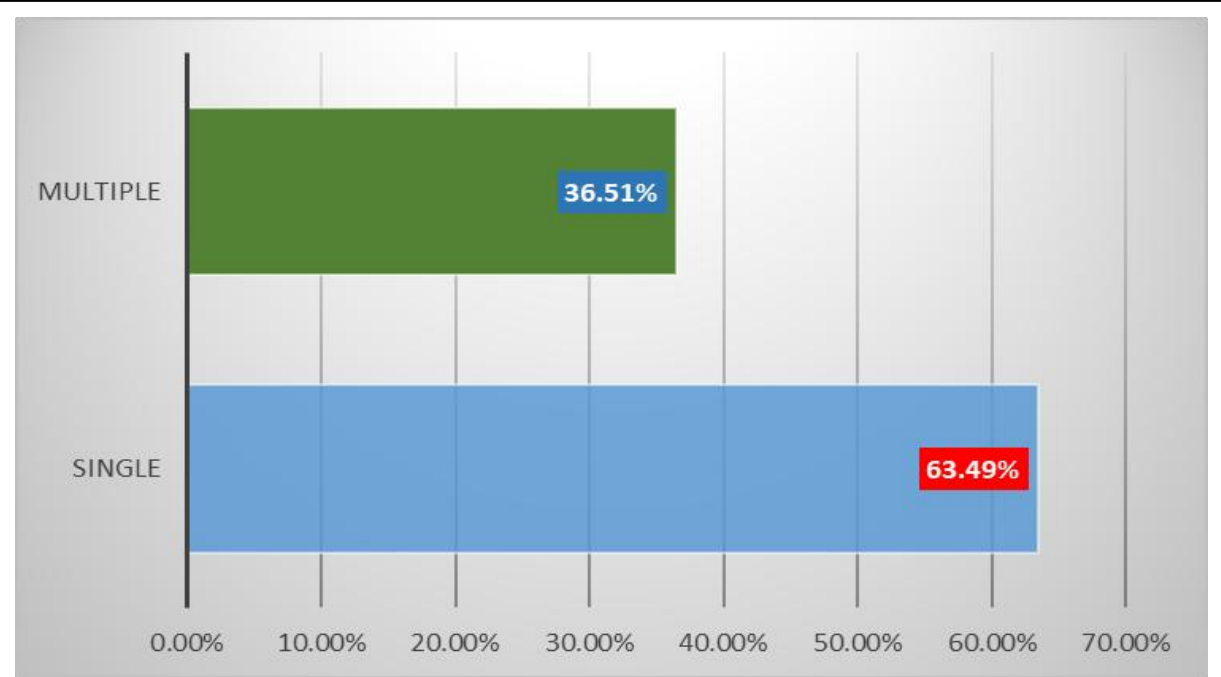

Figure -5: Distribution by Number of Typhoid Ulcer Perforation

In Table-6 shows Distribution by Operative Procedure where primary repair were done In 21 $(33.33 \%)$ patients; repair and ileostomy were done in $21(33.3 \%)$ patients; resection and end to end anastomosis were done in 9 (14.3\%) patients; Table 6: Distribution by Operative Procedure $(n=63)$ resection, anastomosis and proximal ileostomy were done in $12(19.04 \%)$ patients. There is significant association between the various operative modalities $(p<0.05)$. The following table is given below:

\begin{tabular}{|l|c|c|c|}
\hline Operative procedure & Frequency & Percentage & $*$ p \\
\hline Primary repair & 21 & 33.33 & \\
\hline Repair and proximal ileostomy & 21 & 33.33 & \\
\hline Resection and end to end anastomosis & 9 & 14.3 & $<0.05$ \\
\hline Resection; end to end anastomosis and proximal ileostomy & 12 & 19.04 & \\
\hline Total & 63 & 100 & \\
\hline
\end{tabular}

In figure-7 shows Distribution of Postoperative Complication according to Types of Operation where pulmonary infection occurred in 11 patients, septicaemia in 9 patients, renal failure in 3 patients, burst abdomen in 12 patients, pelvic abscess in 9 patients and anastomotic leakage occurred in 7 patients. There is significant association between the patients who had the various types of complications. $(p<0.05)$.

Frequency of complication exceeds total number of patient because same patient developed more than one complication. The following figure is given below:

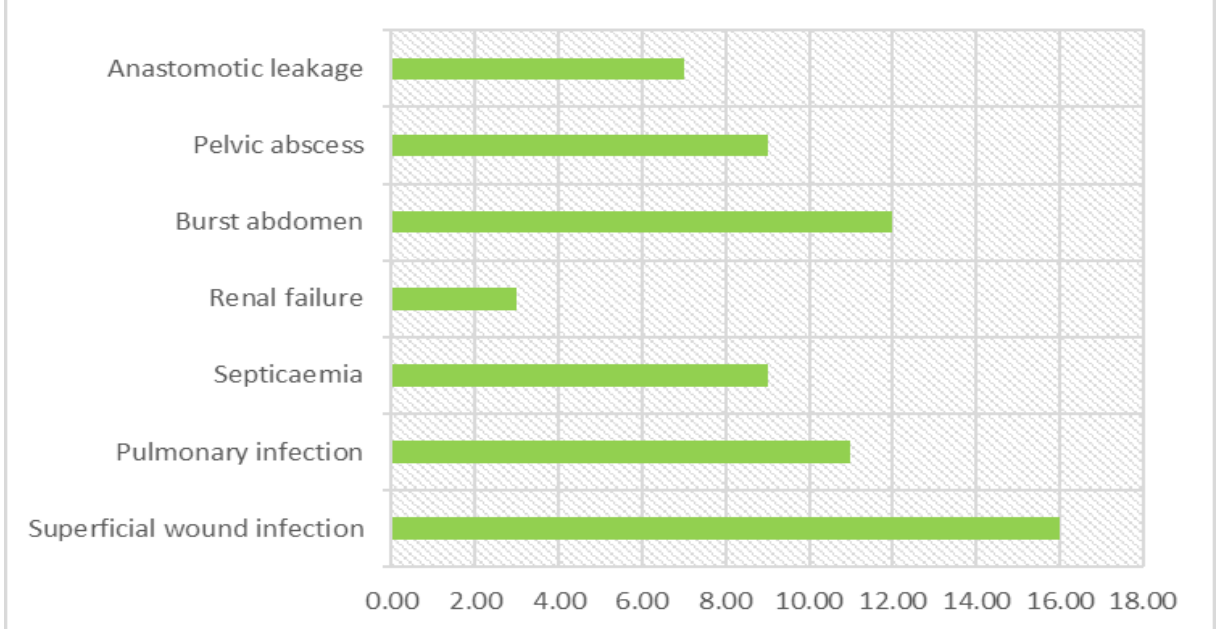

Figure-7: Distribution of Postoperative Complication according to Types of Operation 
In table-7 shows Distribution of complications where 30 patients had got complications. Among them 12 patients had primary repair, 6 patients had primary repair and proximal ileostomy, 10 patients had resection and end to end anastomosis, lastly 2 patients had resection, end to end anastomosis and proximal ileostomy. 8 patients of primary repair; 15 patients of primary repair and proximal ileostomy; 3 patients of resection and end to end anastomosis and 7 patients of resection, end to end anastomosis and proximal ileostomy had no complication. The following table is given below:

Table-7: Distribution of Postoperative Complications According to their Time Lapse before Hospitalization Irrespective of Operation.

\begin{tabular}{|l|l|l|l|}
\hline Complications & $<\mathbf{4 8}$ hours $(\%)$ & $>\mathbf{4 8}$ hours $(\%)$ & *p \\
\hline Wound infection & $1(14.28)$ & $2(9.09)$ & \\
Pulmonary infection & $1(14.28)$ & $1(4.54)$ & \\
Septicaemia & $1(14.28)$ & $3(13.64)$ & \\
Burst abdomen & $1(14.28)$ & $5(22.73)$ & \\
Renal failure & $0(0)$ & $3(13.64)$ & \multirow{2}{*}{} \\
Anastomotic leakage & $1(14.28)$ & $3(13.64)$ & \\
Pelvic abscess & $2(28.56)$ & $5(22.73)$ & \\
\hline Total & $7(100)$ & $22(100)$ & \\
\hline
\end{tabular}

In figure-8 shows Distribution of Post-Operative Mortality according to Time Lapse before Hospitalization Irrespective of Type of Operation where Post-operative death occurred in 2 (25\%) patients who came to the hospital within 48 hours and $6(75 \%)$ patients who came to the hospital after 48 hours of onset of symptoms. There is significant difference between the death of the patients who reached earlier in the hospital and who are not $(\mathrm{p}<0.05)$. The following figure is given below:

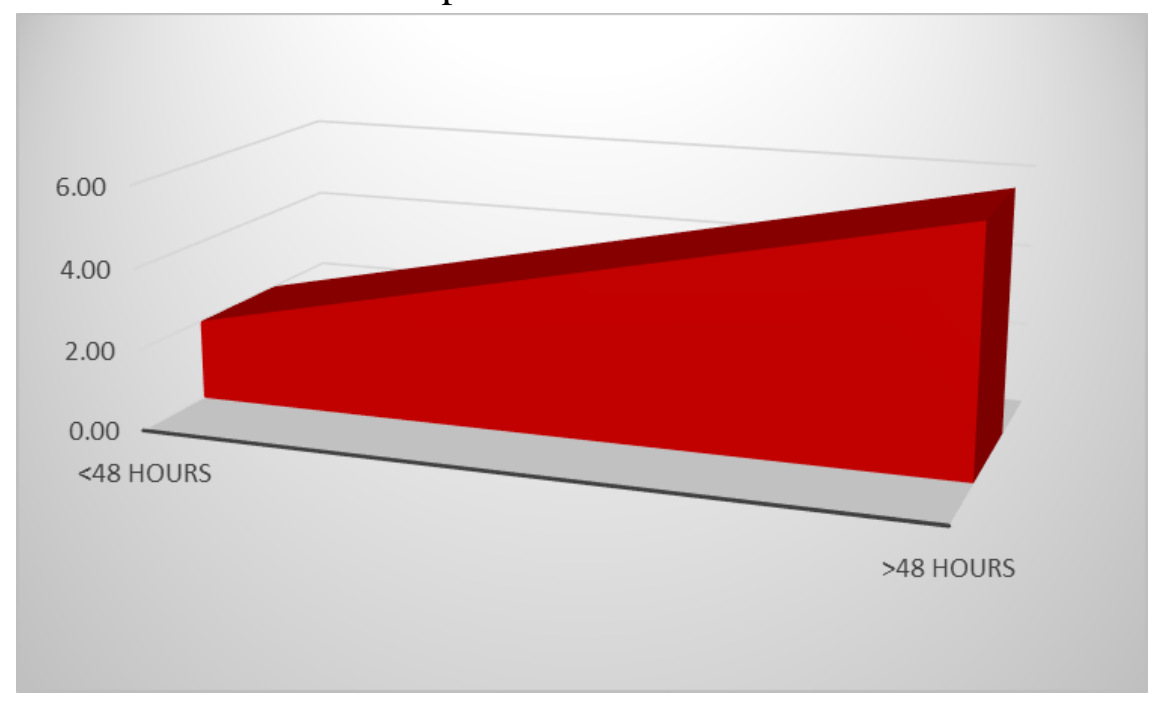

Figure-8: Distribution of Post-Operative Mortality according to Time Lapse before Hospitalization Irrespective of Type of Operation

In table- 8 shows Distribution of Postoperative Mortality According to Types of Operation where Among the 8 patients who are expired in postoperative period $3(37.5 \%)$ patients had primary repair and $5(62.5 \%)$ patients had resection; end to end anastomosis. No patient died who had primary repair with proximal ileostomy and who had resection, end to end anastomosis and proximal ileostomy. 
Table 8: Postoperative Mortality According to Types of Operation

\begin{tabular}{|lc|}
\hline Types of operation & Frequency $(\%)$ \\
\hline Primary repair & $\mathbf{3}(\mathbf{3 7 . 5})$ \\
\hline Primary repair with proximal ileostomy & $\mathbf{0}$ \\
\hline Resection \& end to end anastomosis & $\mathbf{5}(\mathbf{6 2 . 5})$ \\
\hline Resection, end to end anastomosis \& proximal ileostomy & $\mathbf{0}$ \\
\hline Total & $\mathbf{8}$ \\
\hline
\end{tabular}

\section{Discussion}

In this study, the age of the patients ranged from 18 to 80 years with the mean age of 38.6 (SD 14.0) years. This result was supported by Afridi et al. (2008) that the mean age of the patients with perforation peritonitis was 40.5 years (ranges from 13-80 years) and standard deviation was 15.6. Out of 63 typhoid ulcer patients $4(6.35 \%)$ patients were below the age of 20 years. The mean age of this group of patients was $18.76( \pm 0.39) .32$ $(50.79 \%)$ patients were found between the ages of $20-40$ years. Mean age of this group of patients was $32.17( \pm 7.63)$. There were $23(36.51 \%)$ patients in between the age of $40-60$ years. Mean age of this group of patients was $49.11( \pm 8.16) .4$ $(6.35 \%)$ patients were in between the age of $60-$ 80 years. Mean age of this group of patients was $68.39( \pm 1.97)$. There were total 63 patients. Among them 48 (76.19\%) patients were male and $15(23.81 \%)$ patients were female. The ratio of male and female patients was 3.2:1. Ultrasonography findings shows that $26(41.27 \%)$ patients had intraperitoneal collection. The peritoneal cavities of $37(68.73 \%)$ patients were free of intraperitoneal collection. Among the 63 patients 59 patients had leucocytosis and 4 patients had leucopenia. On blood culture 29 patients had positive blood culture and 34 patients had blood culture. 30 patients had found widal test positive and 33 patients had widal test negative. In this study, peritoneal exudates were purulent in 33 $(52.78 \%)$ patients. Fibrinous exudates were found in peritoneal cavity of $26(41.27 \%)$ patients. In 4 $(6.35 \%)$ patients peritoneal cavity was full of serosanguinous fluid. In the current study, among the 63 patients; $40(63.49 \%)$ patients had single perforation and $23(36.51 \%)$ patients had multiple perforations. Ratio of patients of single and multiple perforations was $1.74: 1$. Due to scarcity of literature in this regard these findings could not be compared with the other studies. In this series primary repair were done In 21 (33.33\%) patients; repair and ileostomy were done in 21 (33.3\%) patients; resection and end to end anastomosis were done in $9(14.3 \%)$ patients; resection, anastomosis and proximal ileostomy were done in $12(19.04 \%)$ patients. There is significant association between the various operative modalities $(\mathrm{p}<0.05)$. Other studies also used different types of operations for small bowel perforation $^{[6]}$ Regarding the complication the present study showed Superficial wound infection occurred in 16 (25.39\%) patients, pulmonary infection occurred in $11(17.46 \%)$ patients, septicaemia in $9(14.28 \%)$ patients, renal failure in $3(4.75 \%)$ patients, burst abdomen in $12(19.05 \%)$ patients, pelvic abscess in $9(14.28 \%)$ patients and anastomotic leakage occurred in 7 (11.11\%) patients. There is significant association between the patients who had the various types of complications. $(p<0.05)$. Among the patients who attended hospital within 48 hours of perforation; 1 (14.28\%) patient had wound infection, 1 (14.28\%) had pulmonary infection, 1 (14.28\%) developed septicaemia, 1 (14.28\%) patients had burst abdomen, 1 (14.28\%) had anastomotic leakage and $2(28.57 \%)$ patients developed pelvic abscess. Among the patients who attended hospital after 48 hours of perforation; 2 (9.09\%) patients had wound infection, 1 (4.54\%) had pulmonary infection, 3 $(13.64 \%)$ developed septicaemia, 5 (22.73\%) patients had burst abdomen, 3 (13.64\%) had renal failure, $3(13.64 \%)$ had anastomotic leakage and 5 $(22.73 \%)$ patients developed pelvic abscess. There is significant difference of complications of the 
patients who present in the hospital within 48 hours and after 48 hours $(\mathrm{p}<0.05)$. Post-operative death occurred in 2 (25\%) patients who came to the hospital within 48 hours and $6(75 \%)$ patients who came to the hospital after 48 hours of onset of symptoms. There is significant difference between the death of the patients who reached earlier in the hospital and who are not $(\mathrm{p}<0.05)$. Among the 8 patients who are expired in postoperative period $3(37.5 \%)$ patients had primary repair and $5(62.5 \%)$ patients had resection; end to end anastomosis. No patient died who had primary repair with proximal ileostomy and who had resection, end to end anastomosis and proximal ileostomy.

\section{Conclusion}

After many analysis and examination we can conclude that In case of early presentation - repair of perforation with proximal ileostomy and in case of late presentation - resection, end to end anastomosis with proximal ileostomy have the better outcome. Need large sample size and early diagnosis and treatment of typhoid ulcer perforation to reduce morbidity and mortality.

\section{Reference}

1. Domino, Frank J.; Baldor, Robert A. (2013). The 5-Minute Clinical Consult 2014. Lippincott Williams \& Wilkins. p. 1086. ISBN 9781451188509. Archived from the original on 17 August 2016. Retrieved 4 August 2016.

2. Langell, JT; Mulvihill, SJ (May 2008). "Gastrointestinal perforation and the acute abdomen". The Medical clinics of North America. 92 (3): 599-625, viii-ix. doi:10.1016/j.mcna.2007.12.004. PMID 18387378.

3. Ezzat, Rajan Fuad, Hiwa A. Hussein, Trifa Shawkat Baban, Abbas Tahir Rashid, and Khaled Musttafa Abdullah. "Typhoid ulcer causing life-threatening bleeding from Dieulafoy's lesion of the ileum in a sevenyear-old child: a case report." Journal of medical case reports 4, no. 1 (2010): 171.

4. Anna E. Newton (2014). "3 Infectious Diseases Related To Travel". CDC health information for international travel 2014 : the yellow book. ISBN 9780199948499. Archived from the original on 2015-07-02.

5. https://www.google.com/search?rlz=1C1G GRV_enBD783BD783\&biw=1366\&bih=6 $13 \& \mathrm{tbm}=\mathrm{isch} \& \mathrm{sa}=1 \& \mathrm{ei}=\mathrm{qJk} 7 \mathrm{~W} \_\mathrm{z} 7 \mathrm{O} 4 \mathrm{nrv}$ gSZ8beIAQ\&q=typhoid+ulcer+perforatio n\&oq=typh+perforation\&gs_l=img.1.2.0i7 i30k116j0i8i7i30k1.300048.315109.0.3188 57.7.6.1.0.0.0.181.878.0j6.6.0...0...1c.1.64 .img..0.6.774...0.0.CuEkIABBEew\#imgrc $=$ LjYiEBwTTxleLM:

6. Jhobta, R.S., Attri, A.K., Kaushik, R., Sharma, R., Jhobta, A., 2006. Spectrum of perforation peritonitis in India--review of 504 consecutive cases. World J Emerg Surg, 1, 26. 